\title{
Editorial
}

\section{Perinatal care in Sri Lanka - the past, present and future}

Sri Lanka presently boasts of impressive perinatal statistics; our Maternal Mortality Ratio (MMR) has reduced from 61 in 1995 to 12.5 per 100,000 live births in 2020 . The Neonatal Mortality Rate (NMR) has also reduced from 25 in 1995 to 6.4 per 1000 live births in 2020. These are significant reductions which are now on par with those of more developed countries, having a higher per capita income than ours. The reasons of this success story are multifactorial; A firm foundation and network of community health care services, along with free universal health care and education, improved female literacy rates and firm political commitment by successive governments have all contributed to these achievements.

Even in the 1990's the majority of births occurred in hospitals, but the maternal and neonatal mortality rates were high. The major causes of maternal mortality then were post partumhaemorrhage and maternal sepsis, while neonates died mainly due to congenital anomalies, prematurity, and sepsis, in that order. There was no hope for extremely preterm babies. While maternal care was already fairly well established during this era, the neonatal care was rather rudimentary. In the larger hospitals, the Premature Baby Units(PBU) consisted of a nursery with some cots and a couple of incubators, while in the smaller institutions the latter was substituted by improvised "boxes" with lights fixed as warming devises. Ventilation for sick neonates was almost nonexistent, while many babies died of neonatal tetanus. The mainstay of neonatal care was basic incubator care, intravenous fluids and available antibiotics. Beyond this it was a matter of "survival of the fittest".

Sri Lanka has come a long way since then; The main causes of maternal mortality now are post partumhaemorrhage and heart disease while in neonates it is congenital anomalies, prematurity and sepsis.

Maternal care has improved greatly; over 95\% mothers receive complete antenatal care and nearly $90 \%$ of deliveries occur in hospitals with specialist cover. However, preventable causes of maternal deaths such as post partumhaemorrhage (which should only rarely occur in a hospital setting), still remain as a major cause. Heart disease in pregnancy is another cause where prepregnancy counseling appears to have failed; these are mothers who should not have become pregnant in the first place. Maternal suicides are still not accounted for in calculating the Maternal Mortality Ratio. If this were the case, the mortality figures would most certainly increase; rather than be complacent with the present figures, these statistics will be vital to improving mental health for mothers.

Over the last few decades, neonatal care has also improved tremendously; neonatal tetanus and congenital rubella have been eliminated due to the success of the Expanded Immunization Programme (EPI). Survival of extremely preterm neonates are now a reality. For neonates, today we have over 17 fully fledged neonatal intensive care units (NICU's) in the country offering advanced neonatal care such as high frequency ventilation(HFOV), Nitric Oxide $\left(\mathrm{NO}_{2}\right)$ 
therapy for pulmonary hypertension and total body cooling for term babies with severe birth asphyxia, the latter two being the results of pioneering efforts by members of the perinatal society. Many of these units are now manned by specially trained neonatologists who are well versed in these advanced techniques. These as well as other advances in NICU care such as the availability of Surfactant for premature infants, use of newer technology and equipment and successful promotion of exclusive breast feeding has brought down the neonatal mortality figures to the present status.

Sri Lanka has now reached a milestone in perinatal care, with maximum gained from utilizing cost effective methods. Hence we now need to emphasize on improving quality of care for both mother and baby; For mothers reducing, preventable maternal deaths should be a priority while finer issues such as respectful labor and mental health should be addressed. In neonatal care, we have now reached the "last mile" in our quest, which proverbially is the hardest. If we are to improve further, we need to invest on strengthening our NICU care; measures to improve survival and outcome of extreme preterm babies such as providing Total Parenteral Nutrition (TPN), should be addressed. Not only survival, but ensuring a good quality of life for the NICU survivors should be of utmost importance. Providing formal training in NICU care for both medical officers and nurses should be prioritized. This issue is being addressed at present by the Perinatal Society, with the ongoing development of an NICU training module for doctors and nurses working in NICU's of the country.

The Sustainable Development Goals (SDG) require us to reduce our MMR and NMR to less than 10 and 2.2 respectively by 2030 . Therefore we now have to take perinatal care to the next level, by investing wisely and guiding the pathway to maternal and newborn care for the future.

Prof Dulanie Gunasekera

Editor in Chief 